\title{
Justifying the Special Theory of Relativity with Unconceived Methods
}

\begin{abstract}
Many realists argue that present scientific theories will not follow the fate of past scientific theories because the former are more successful than the latter. Critics object that realists need to show that present theories have reached the level of success that warrants their truth. I reply that the special theory of relativity has been repeatedly reinforced by unconceived scientific methods, so it will be reinforced by infinitely many unconceived scientific methods. This argument for the special theory of relativity overcomes the critics' objection, and has advantages over the no-miracle argument and the selective induction for it.
\end{abstract}

\section{Keywords}

Pessimistic Induction, Scientific Realism, Special Theory of Relativity, Unconceived Methods

Park, Seungbae (2018). "Justifying the Special Theory of Relativity with Unconceived Methods", Axiomathes 28 (1): 53-62.

Seungbae Park

Ulsan National Institute of Science and Technology

Republic of Korea

nature@unist.ac.kr

\section{Introduction}

Henri Poincaré (1905/1952: 160), Ernst Mach (1911: 17), Larry Laudan (1977: 126), Hilary Putnam (1978: 25), P. Kyle Stanford (2006: 19-20), and K. Brad Wray (2013: 4327) formulated the pessimistic induction that since past theories were overturned, present theories will also be overturned. John Worrall (1989: 99), Philip Kitcher (1993: 136), Stathis Psillos (1999), P. D. Magnus and Craig Callender (2004: 322), and Wray (2013: 4321) regard the pessimistic induction as the strongest objection to scientific realism. In response to the pessimistic induction, Jarrett Leplin (1997: 141), Gerald Doppelt (2007: 111; 2014), Juha Saatsi (2009: 358), Michael Devitt (2011: 292), Ludwig Fahrbach (2011: 1290), Seungbae Park (2011: 80), and Moti Mizrahi (2013) point out that present theories are more successful than past theories were, so it is wrong to project the fate of past theories onto present theories.

Florian Müller (2015) and Mario Alai (2016) admit that present theories have higher explanatory and predictive powers than past theories. They argue, however, that the mere superiority of present theories over past theories does not establish realism. Müller says that it "is not at all obvious why science, or at least our current best theories, should have achieved a degree of success that warrants their truth" (2015: 406). Alai claims that it "is hard to think that any improvement of our background empirical knowledge and methods can at some point make scientists practically infallible; and even more that this point has already been reached" (2016: 16). Meeting these two philosophers' challenge to realism requires more than refuting the pessimistic induction. It requires constructing a positive argument for realism.

This paper aims to construct such an argument for the special theory of relativity by ironically making use of the very theoretical resources that pessimists (Stanford, 2006; Wray, 2016) have developed to attack realism. I proceed as follows. In Section 2, I explicate Wray's (2016) argument from unconceived methods according to which some present theories will be refuted by hitherto unconceived methods, just as some past theories were refuted by the 
then unconceived methods. ${ }^{1}$ In Section 3, I provide some examples in which new data generated by previously unconceived methods strengthened rather than weakened accepted theories. In Section 4, I argue that since the special theory of relativity has been repeatedly reinforced by new methods, it will continue to be reinforced by new methods indefinitely into the future. In Section 5, I explain how this argument for the special theory of relativity gets around Wray's argument from unconceived methods, and Müller's and Alai's challenge to realism stated above. In addition, I delineate the advantages of the argument for the special theory of relativity over the no-miracle argument and the selective induction for it.

\section{The Argument from Unconceived Methods}

What is an unconceived method? To use Wray's (2016: 369) example, a telescope was an unconceived method when the geocentric system was put forward in the second century. Galileo invented one in the seventeenth century, and then observed the phases of Venus and the moons of Jupiter with it, thereby refuting the geocentric system. So an unconceived method can be defined as a method that is unconceived at a certain point in time, but is conceived at a later point in time, generating new data that are qualitatively different from old data.

Now that the concept of an unconceived method is clear, we are ready to unpack Wray's argument from unconceived methods. Wray admits that present theories are more successful than past theories due to technological developments. He argues, however, that new methods generate new data, and that "some of these new data will require us to accept a radically new theory" (2016: 372). He also says that "some of the changes of theory in the past have been due to changes in methodology, and some of the changes of theory in the future will also likely be due to changes in methodology" (2016: 372-373). So the argument from unconceived methods comes down to the induction that just as some past theories were overthrown by unconceived methods, so some present theories will be overthrown by unconceived methods. Note that it does not assert that just as most past theories were overturned by unconceived methods, so most present theories will be overturned by unconceived methods.

Why should we accept the premise that some past theories were discarded by unconceived methods? Wray (2016: 370-372) provides the following three examples to justify it:

(1) DNA sequencing was unconceived at the end of the nineteenth century, when the remains of Neanderthals were discovered. Anthropologists thought that these remains were of homo sapiens with a pathological condition. In the early twenty-first century, however, DNA sequencing revealed that Europeans and Asians inherited some genes from Neanderthals, but Africans did not.

(2) Oceanography disclosed the magnetic reversals on the ocean floor in the midtwentieth century, thereby contributing to the advent of the theory of plate tectonics.

(3) Planck employed probability theory to solve the black-body problem. He discovered that the colors of a heated body are correlated with different temperatures. This discovery led to the demise of classical mechanics and to the rise of quantum mechanics.

\footnotetext{
1 In this paper, 'some' expresses a smaller quantity than 'most.'
} 
Do these three examples suffice to establish the premise of the argument from unconceived methods? My answer is yes. Recall that the premise states not that most past theories were ousted by unconceived methods but that some past theories were ousted by unconceived methods. The three examples establish the latter, although not the former. Establishing the former requires going through a random sampling process. Pessimists should randomly choose some theories from the population of past theories, and then check whether each of the selected theories was refuted by an unconceived method. If most of the sample theories were refuted by unconceived methods, pessimists are entitled to infer that most past theories were refuted by unconceived methods.

The argument from unconceived methods is compatible with realism, the view that most successful theories are (approximately) true. ${ }^{2}$ Realism does not assert that all successful theories are true. For example, Putnam says that "the theories accepted in a mature science are typically approximately true" (1975: 73). Note that he says not that mature scientific theories are definitely true but that they are typically true. Also, Devitt says that "[m]ost of the essential unobservables of well-established current scientific theories exist mindindependently" (2011: 286). Note that he says not that all of the essential unobservables exist but that most of the essential unobservables exist. Realism, thus defined, does not contradict the argument from unconceived methods which asserts that some past and present theories are discarded by unconceived methods.

In this sense, the argument from unconceived methods is different from Stanford's (2006: 19-20) problem of unconceived alternatives, which states that most present theories will be replaced by unconceived alternatives, as were most past theories. Stanford says that "the history of scientific inquiry itself offers a straightforward rationale for thinking that there typically are alternatives to our best theories" (2006: 20). Note that he uses the qualifier 'typically,' which implies that most past theories were replaced by unconceived alternatives. Therefore, Stanford's problem of unconceived alternatives is much stronger, and hence more intriguing, than Wray's argument from unconceived methods.

\section{Strengthen}

Admittedly, some technological developments do weaken accepted theories. Other technological developments, however, rather strengthen them. Let me provide three such examples:

- A telescope was unconceived in the sixteenth century when Copernicus put forward the heliocentric system. Galileo invented one in the seventeenth century, and produced new data, providing an additional justification for the heliocentric system.

- An electron microscope was unconceived at the end of the nineteenth century when the germ theory of diseases was accepted. It was developed in the twentieth century and strengthened the germ theory of diseases.

- The Hubble telescope was unconceived in 1915, when Albert Einstein advanced the general theory of relativity, according to which gravitational lensing exists. Scientists later observed gravitational lensing with the Hubble telescope, thereby reinforcing the general theory of relativity (van der Well et al., 2013).

${ }^{2}$ I drop the qualifier 'approximately' for the sake of simplicity in this paper. 
These three examples show that the population of unconceived methods is composed of those that strengthen accepted theories as well as those that weaken accepted theories. So if we randomly select some sample methods from the population, some of them will be those that strengthen existing theories, and others will be those that weaken existing theories. If you selected only the former or only the latter, your sample is biased.

Which position are unconceived methods favorable to, realism or pessimism? The answer to this question depends on the number of unconceived methods that strengthen accepted theories, compared with that of unconceived methods that weaken accepted theories. If the former is greater than the latter, unconceived methods are overall favorable to realism; if the latter is greater than the former, unconceived methods are overall favorable to pessimism. Thus, to determine whether unconceived methods are overall favorable to realism or pessimism, we should randomly select some methods from the population of methods that were unconceived at certain points in time, but were later conceived, and then should check whether each sample method strengthened or weakened an accepted theory, which would be a grueling task. That does not mean, however, that the prospect for realism is dim. In the next section, I defend a version of realism vis-à-vis unconceived methods.

\section{The Optimistic Argument from Unconceived Methods}

In 1905, Einstein proposed the special theory of relativity, according to which time for a moving object flows more slowly than for a stationary object, and as an object moves faster, it gains mass. New methods have repeatedly bolstered this theory, including the following three methods:

\section{The List of Unconceived Methods}

- Fast-moving jets and atomic clocks were unconceived in 1905. In 1971, a fastmoving jet carrying four atomic clocks flew around the world twice, and then the atomic clocks were compared with those on the ground. Time dilation was ascertained (Hafele and Keating, 1972).

- Particle accelerators were unconceived in 1905. They began to be built in the midtwentieth century. When a particle is accelerated near to the speed of light, the increase in its kinetic energy can be precisely measured, and this increase in kinetic energy confirms the increase in the particle's mass (Plettner, Byer, and Siemann, 2005).

- The global positioning system (GPS) was unconceived in 1905 and was developed in the second half of the twentieth century. It uses the special theory of relativity and the general theory of relativity to accurately determine the location of a GPS receiver. Whenever GPS works, it confirms the two theories.

These three examples constitute an inductive rationale for thinking that there are more hitherto unconceived methods, which will be conceived and will further strengthen the special theory of relativity. As those hitherto unconceived methods bolster the special theory of relativity, our descendents will have a stronger inductive rationale for thinking that the special theory of relativity is true, and that there are more unconceived methods that will provide additional justifications for the special theory of relativity. It follows that there are infinitely many such methods. This argument for the special theory of relativity is an example of what I call the optimistic argument from unconceived methods. 
The foregoing optimistic argument concerns only the special theory of relativity. It does not concern other scientific theories, such as the oxygen theory, the kinetic theory, the Big Bang theory, evolutionary theory, and the theory of plate tectonics. In other words, if you want to believe that, say, the oxygen theory is fated to be bolstered by infinitely many unconceived methods, you should provide some examples of previously unconceived methods that were later conceived and have augmented the oxygen theory, and then construct the optimistic argument from unconceived methods for the oxygen theory. The same goes for other scientific theories.

The optimistic argument enshrines the view which I call scientific individualism. It holds that we should evaluate scientific theories individually. It contrasts with what I call scientific collectivism, which holds that we should evaluate scientific theories collectively. Different individualists have different views about a particular scientific theory. Some individualists believe that it is true; other individualists do not believe that it is true. The former are individual realists about it; the latter are individual antirealists about it. The same is true of collectivists. Some collectivists believe that a group of theories is true; other collectivists do not believe that the group of theories is true. The former are collective realists about the group of theories; the latter are collective antirealists about the group of theories. In sum, the unit of evaluation is an individual theory and a set of theories for individualists and for collectivists, respectively.

The optimistic argument mirrors Stanford's problem of unconceived alternatives in certain respects. Stanford provides the following list of past and present theories to justify the problem of unconceived alternatives:

\section{$\underline{\text { Stanford's List }}$}

from elemental to early corpuscularian chemistry to Stahl's phlogiston theory to Lavoisier's oxygen chemistry to Daltonian atomic and contemporary chemistry

from various versions of preformationism to epigenetic theories of embryology

from the caloric theory of heat to later and ultimately contemporary thermodynamic theories

from effluvial theories of electricity and magnetism to theories of the electromagnetic ether and contemporary electromagnetism

from humoral imbalance to miasmatic to contagion and ultimately germ theories of disease

from eighteenth century corpuscular theories of light to nineteenth century wave theories to the contemporary quantum mechanical conception

from Darwin's pangenesis theory of inheritance to Weismann's germ-plasm theory to Mendelian and then contemporary molecular genetics

from Cuvier's theory of functionally integrated and necessarily static biological species and from Lamarck's autogenesis to Darwin's evolutionary theory (Stanford, 2006: 19-20)

Stanford takes this list to provide an inductive rationale for thinking that the possibility space of unconceived alternatives "appears to be indeterminate and unbounded" (2006: 133). In other words, there are infinitely many unconceived alternatives to the kinetic theory, evolutionary theory, the germ theory, electromagnetic theory, and so on. Notice that each item on Stanford's list consists of, at best, several theories. For example, the transitions of theories of diseases consist of the humoral theory, the miasma theory, and the germ theory. 
These three theories, Stanford claims, constitute an inductive rationale for thinking that there are infinitely many theories of diseases. The fact that present scientists cannot conceive of the infinitely many alternatives does not show that there are no infinitely many unconceived alternatives. It only shows that present scientists are similar to past scientists "whose cognitive constitutions are not well suited to the task of exhausting the kinds of spaces of serious candidate theoretical explanations from which our scientific theories are drawn" (Stanford, 2006: 45).

If Stanford's pessimistic induction is correct, the optimistic argument from unconceived methods for the special theory of relativity is also correct. The three new methods of the fast moving jets/atomic clocks, particle accelerators, and GPS constitute an inductive rationale for thinking that there are infinitely many unconceived methods that will fortify the special theory of relativity, just as the three theories of diseases constitute an inductive rationale for thinking that there are infinitely many theories of diseases. The fact that present scientists cannot conceive of the infinitely many methods does not signify that there are no infinitely many unconceived methods. It only signifies that present scientists are similar to past scientists who lacked the cognitive capacity to think up infinitely many methods. There is no reason to think that the pessimistic inference is correct while the optimistic inference is incorrect.

Stanford (2006: 19) claims that unconceived alternatives are not slightly but radically distinct from one another. Wray agrees with Stanford, saying that some present theories will be replaced "with theories that posit radically different entities or ascribe radically different properties" (2016: 369). If Stanford and Wray are right, it is doubtful that present theories are approximately true. Just as Stanford and Wray contend that unconceived alternatives are radically distinct from one another, so I contend that unconceived methods are radically distinct from one another. The aforementioned three unconceived methods that augmented the special theory of relativity are radically distinct from one another. This radical difference between the unconceived methods means that data generated by a new method are qualitatively distinct from those generated by an old method, which in turn means that the additional support that each of infinitely many unconceived methods will provide for the special theory of relativity is not negligible but significant. We are justified in believing that such a theory is true.

\section{Advantages}

The optimistic argument from unconceived methods for the special theory of relativity is immune to some counterexamples that pessimists might be tempted to give. The examples of DNA sequencing, oceanography, and probability theory do not refute the optimistic argument. If pessimists wish to refute the optimistic argument, they should provide examples of unconceived methods that weakened the special theory of relativity, not examples of unconceived methods that weakened other scientific theories. The examples of DNA sequencing, oceanography, and probability theory are simply red herrings in the context of the debate over whether we are justified in believing that the special theory of relativity is true. In a nutshell, the optimistic argument, which embeds scientific individualism, is not undermined at all by Wray's argument from unconceived methods.

The optimistic argument overcomes not only Wray's argument from unconceived methods but also Müller's and Alai's challenge to realism mentioned in the introduction of this paper. Their challenge was that realists need to present a positive argument to show that present theories have reached the level of success that warrants their truth. Realists can construct such an argument as follows. It does not matter whether the special theory of relativity has achieved the degree of success that warrants its truth or not. Even if we grant 
for the sake of argument that the current observational evidence for it only warrants assigning $1 \%$ probability to it, we are justified in believing that it is true. After all, it is fated to be strengthened by infinitely many unconceived methods, so its probability will go above $99 \%$, although it will not go above $100 \%$. There is nothing wrong with believing now that it is true.

The optimistic argument is superior to the no-miracle argument (Putnam, 1975: 73) for the special theory of relativity, ${ }^{3}$ which holds that the success of the special theory of relativity would be a miracle, if it were false. It can make accurate predictions about the time dilation and length contraction of fast-moving objects because it is true. The optimistic argument and the no-miracle argument have the same conclusion that the special theory of relativity is true. But they have different premises. The premise of the no-miracle argument appeals to the finite number of impressive empirical performances that the special theory of relativity has shown so far, while the premise of the optimistic argument appeals to the infinite number of impressive empirical performances that the special theory of relativity has shown and will show. Therefore, the inference from the premise to the conclusion of the optimistic argument is tighter and less risky than that from the premise to the conclusion of the no-miracle argument.

The optimistic argument has another advantage over the no-miracle argument for the special theory of relativity. Unlike the no-miracle argument, the optimistic argument parallels Stanford's problem of unconceived alternatives. Consequently, it is difficult for pessimists to attack the optimistic argument without also attacking the problem of unconceived alternatives, one of the strongest objections to realism. Any criticism that pessimists might launch against the optimistic argument, I suspect, can also be directed at the problem of unconceived alternatives. For example, pessimists might argue that it is fallacious that since the special theory of relativity was augmented by three unconceived methods, it will be augmented by infinitely many unconceived methods. Optimists would retort that it is also fallacious that since there were three theories of diseases, there are infinitely many unconceived theories of diseases.

Let me turn to the advantages of the optimistic argument over the selective induction for the special theory of relativity. The selective induction holds that the special theory of relativity is composed of stable and unstable posits, as was its predecessor. The predecessor's stable posits were preserved in the special theory of relativity, but its unstable posits were discarded. Analogously, stable posits of the special theory of relativity will be carried over to its successor, whatever it might be, while its unstable posits will be abandoned. It follows that only the stable posits are worthy of our beliefs, and that the special theory of relativity is approximately true. Selective realism is endorsed by many eminent philosophers: Worrall (1989), Kitcher (1993: Chapters 4 and 5), Psillos (1999: Chapter 6), Anjan Chakravartty (2008), Patrick Enfield (2008), Peter Godfrey-Smith (2008), David Harker (2008), Psillos (2009), Juha Saatsi (2009), and Samuel Ruhmkorff (2011: 882). Keep in mind that selective realism presupposes, as pessimism does, that the special theory of relativity will be displaced by an unconceived alternative.

Stanford raises the following two objections to selective realism. First, selective realists have not provided a prospectively applicable criterion for distinguishing between stable and unstable posits (Stanford, 2009: 385). In the absence of such a criterion, we cannot know which theoretical components of a present theory merit our doxastic commitments, and which theoretical components do not. Second, given that past and present theories are radically distinct from one another, present and future theories will also be radically distinct from one another, and hence it is merely a matter of taste whether to attribute the predicate

\footnotetext{
3 Putnam's no-miracles argument ranges over all successful theories.
} 
'approximately true' to present theories. In other words, the difference between pessimists and selective realists "is simply a difference of style or taste in applying the expression 'approximately true' rather than a substantive disagreement between them" (Stanford, 2015: 876). This objection to selective realism implies that 'selectivism' is a better nomenclature than 'selective realism.'

Stanford's preceding two criticisms against selectivism do not apply to the optimistic argument. On the optimist account, no scientific revolution will oust the special theory of relativity, and the special theory of relativity will only be augmented by infinitely many unconceived methods. Hence, there is no need to distinguish between stable and unstable posits of the special theory of relativity, there is a substantive disagreement between optimists and pessimists, and optimists deserve the label 'realists' while selectivists do not.

\section{Conclusion}

Stanford's problem of unconceived alternatives and Wray's argument from unconceived methods are intended to rebut realism. They contain valuable insights that can be utilized to construct the optimistic argument from unconceived methods for a particular scientific theory. If a particular scientific theory was strengthened by a few of unconceived methods, we can construct an optimistic argument that since it received a significant additional confirmation from each of the unconceived methods, it will receive a significant additional confirmation from each of infinitely many hitherto unconceived methods. This optimistic argument gets around the challenges posed by Müller, Wray, and Alai. It also has advantages over the nomiracle argument and the selective induction for a particular theory. This paper can be summed up in a simple slogan: unconceived methods are realists' friends.

\section{References}

Alai, Mario (2016). "Resisting the Historical Objections to Realism: Is Doppelt's a Viable Solution?" Synthese. doi:10.1007/s11229-016-1087-z.

Chakravartty, Anjan (2008). "What You Don't Know Can't Hurt You: Realism and the Unconceived", Philosophical Studies 137 (1): 149-158.

Devitt, Michael (2011). “Are Unconceived Alternatives a Problem for Scientific Realism?", Journal for General Philosophy of Science 42 (2): 285-293.

Doppelt, Gerald. (2007). "Reconstructing Scientific Realism to Rebut the Pessimistic Metainduction", Philosophy of Science 74 (1): 96-118.

--------- (2014). “Best Theory Scientific Realism”, European Journal for Philosophy of Science 4 (2): 271-291.

Enfield, Patrick (2008). "P. Kyle Stanford, Exceeding Our Grasp: Science, History, and the Problem of Unconceived Alternatives", British Journal for the Philosophy of Science 59 (4): 881-895.

Fahrbach, Ludwig (2011). "Theory Change and Degrees of Success", Philosophy of Science 78 (5): 1283-1292.

Godfrey-Smith, Peter (2008). "Recurrent Transient Underdetermination and the Glass Half Full”, Philosophical Studies 137: 141-148. 
Hafele, Joseph C. and Keating, Richard E. (July, 1972). "Around-the-World Atomic Clocks: Predicted Relativistic Time Gains”, Science 177 (4044): 166-168.

Harker, David (2008). "P. Kyle Stanford, Exceeding Our Grasp: Science, History, and the Problem of Unconceived Alternatives", Philosophy of Science 75 (2): 251-253.

Kitcher, Philip (1993). The Advancement of Science: Science without Legend, Objectivity without Illusions. New York: Oxford University Press.

Laudan, Larry (1977). Progress and Its Problems: Towards a Theory of Scientific Growth. California: University of California Press.

Leplin, Jarrett (1997). A Novel Defense of Scientific Realism. New York: Oxford University Press.

Mach, Ernst (1911). History and Root of the Principle of the Conservation of Energy (Jourdain P. E. B., Trans.). Chicago: Open Court Publishing Company.

Magnus, P. D. and Craig Callender (2004). "Realist Ennui and the Base Rate Fallacy", Philosophy of Science 71 (3): 320-338.

Mizrahi, Moti (2013). "The Pessimistic Induction: A Bad Argument Gone Too Far", Synthese 190 (15): 3209-3226.

Müller, Florian (2015). "The Pessimistic Meta-induction: Obsolete Through Scientific Progress?", International Studies in the Philosophy of Science 29 (4): 393-412.

Park, Seungbae (2011). "A Confutation of the Pessimistic Induction", Journal for General Philosophy of Science 42 (1): 75-84.

Plettner, Thomas, Robert L. Byer, and Robert H. Siemann (2005). “The Impact of Einstein's Theory of Special Relativity on Particle Accelerators", Journal of Physics B: Atomic, Molecular and Optical Physics 38: S741-S752.

Poincaré, Henri (1905/1952). Science and Hypothesis. New York: Dover.

Psillos, Stathis (1999). Scientific Realism: How Science Tracks Truth. New York: Routledge.

--------- (2009). “Grasping at Realist Straws”, Review Symposium, Metascience 18: 363-370.

Putnam, Hilary (1975). Mathematics, Matter and Method (Philosophical Papers, vo. 1), Cambridge: Cambridge University Press.

(1978). Meaning and the Moral Sciences. London: Routledge \& K. Paul.

Ruhmkorff, Samuel (2011). "Some Difficulties for the Problem of Unconceived Alternative", Philosophy of Science 78 (5): 875-886. 
Saatsi, Juha (2009). "Grasping at Realist Straws”, Review Symposium, Metascience 18: 355362.

Stanford, P. Kyle (2006). Exceeding Our Grasp: Science, History, and the Problem of Unconceived Alternatives. Oxford: Oxford University Press.

-------- (2009). “Grasping at Realist Straws”, Review Symposium, Metascience 18 (3): 379390.

(2015). "Catastrophism, Uniformitarianism, and a Scientific Realism Debate That Makes a Difference”, Philosophy of Science 82 (5): 867-878.

van der Well, A., G. van de Ven, M. Maseda, H.W. Rix, G.H. Rudnick, A. Grazian, S.L. Finkelstein, D.C. Koo, S.M. Faber, H.C. Ferguson, A.M. Koekernoer, N.A. Grogin, D.D. Kocevski (2013). "Discovery of a Quadruple Lens in CANDELS with a Record Lens Red shift Z=1.53”, Astrophysical Journal Letters. arXiv:1309.2826.

Worrall, John (1989). "Structural Realism: The Best of Both Worlds", Dialectica 43 (1-2): 99-124.

Wray, K. Brad (2013). "Pessimistic Induction and the Exponential Growth of Science Reassessed", Synthese 190 (18): 4321-4330.

(2016). "Method and Continuity in Science", Journal for General Philosophy of Science 47 (2): 363-375. 